\title{
Self-aggregation of poly(methyl methacrylate)-block-poly(sulfonated glycidyl methacrylate) copolymers
}

\author{
Jean-François Gohy, Sayed Antoun and Robert Jérôme
}

Center for Education and Research on Macromolecules (CERM), Institute of Chemistry B6, University of Liège, Sart-Tilman, B-4000 Liège, Belgium

\begin{abstract}
The self-aggregation of a series of amphiphilic poly(methyl methacrylate)-blockpoly(sulfonated glycidyl methacrylate) (PMMA- $b$-PSGMA) copolymers in water has been studied by dynamic light scattering (DLS), size exclusion chromatography (SEC) and surface tension measurements. All the copolymers considered in this study form micelles which, however, aggregate into larger structures as shown by DLS and SEC. These larger structures dissociate into smaller ones upon increasing the temperature. The extent of this dissociation process is strongly dependent on the copolymer composition. It is large as the content of the hydrophilic block is high.
\end{abstract}

Author Keywords: Self-aggregation; Block copolymer; Micelles

\section{Introduction}

Formation of polymeric micelles in aqueous media deserves interest, because of potential applications in various fields, such as drug carriers [1-2], dispersion of pigments in paints [3], and stabilization of latex particles [4]. Dissolved in water, amphiphilic copolymers form micelles, that consist of a core of the insoluble blocks surrounded by a shell of the watersoluble blocks [5-8].

Some of the most extensively investigated amphiphiles include block copolymers of ethylene oxide and butylene oxide [9-11], ethylene oxide and propylene oxide [12-20], ethylene oxide and styrene [21-22], quaternized 4-vinylpyridine and styrene [23-28], methacrylic acid and styrene [29-35] and methylmethacrylate and dimethylaminoethylmethacrylate [36-38]. Micellization of amphiphilic copolymers in water was reviewed in 1985 by Selb and Gallot [39]. Although, spherical micelles were then reported, rod-like micelles, lamellae, vesicles, and tubules have been observed more recently [40-43]. Eisenberg et al. have reported on the formation of the so-called 'crew-cut' micelles, formed by highly asymmetric diblock copolymers consisting of a short hydrophilic polyelectrolyte or neutral block and a long hydrophobic polystyrene or polybutadiene block [40;42]. These micelles are formed by the addition of a small amount of water to the copolymer dissolved in a non-selective solvent, e.g. dimethylformamide. They are kinetically frozen in by adding a large excess of water followed by the removal of the non-selective solvent by dialysis. The amphiphilic copolymers investigated in this study have a completely different composition since the hydrophilic block is the major constituent. Poly(acrylic acid) and poly(methacrylic acid) blocks are commonly used as precursors of anionic blocks. The ionization of these weak polyacids is, however, $\mathrm{pH}$ dependent which causes modification in the chain solvation and in the stabilization of the micelles. In this study, a strong poly(sulfonic acid) block has been used, because the parent anionic polyelectrolyte block is insensitive to $\mathrm{pH}$. Till now, poly(sulfonated glycidyl methacrylate) (PSGMA) has been rarely studied as a stabilizing block in polymeric micelles, although this polyanion can be easily derivatized from poly(glycidyl methacrylate) (PGMA). 
Moreover, block copolymers of GMA and various alkyl methacrylates can be prepared by sequential living anionic polymerization of the comonomers [44]. Poly(alkyl methacrylate)block-poly(sulfonated glycidyl methacrylate) diblocks have been used to stabilize efficiently very fine polyacrylic lattices in aqueous medium [45]. High critical coagulation concentrations have been reported for these lattices as result of the high efficiency of the PSGMA electrosteric barrier. More recently, the kinetics of adsorption of a poly(tert-butyl methacrylate)-block-poly(sulfonated glycidyl methacrylate) diblock on hydrophobic substrate has been investigated [46] and the interactions between these coated surfaces have been estimated by measurement of the surface forces [47]. Long-range electrostatic repulsive interactions have been observed in relation to the large stretching of the PSGMA chains in salt-free aqueous solution.

From these few studies, it is clear that PSGMA-containing amphiphilic copolymers have great potential in various applications. However, the self-association in water has not been investigated in detail, which is the purpose of this paper in the case of poly(methyl methacrylate)-block-poly(sulfonated glycidyl methacrylate) (PMMA-b-PSGMA) copolymers.

\section{Experimental}

\subsection{Copolymer synthesis}

The diblock copolymers were anionically synthesized as reported elsewhere [44]. Briefly, the monomers were purified by distillation over triethylaluminum. The glass reactor containing the required amount of $\mathrm{LiCl}(10 / 1 \mathrm{LiCl} /$ initiator molar ratio) was flamed dried under vacuum, purged with nitrogen, added with the solvent (tetrahydrofuran previously dried by reflux over sodium) and cooled down to $-78^{\circ} \mathrm{C}$. Diphenylmethyllithium was added dropwise until a persistent orange color was observed, followed by the required amount of this initiator. Methylmethacrylate was first polymerized at $-78^{\circ} \mathrm{C}$ for $1 \mathrm{~h}$, followed by GMA $(2 \mathrm{~h}$ at $-78^{\circ} \mathrm{C}$ ). A sample was picked out from the reactor before the addition of GMA. The copolymerization reaction was then quenched by addition of methanol.

A star-shaped block copolymer was synthesized by the method reported by Rempp and coworkers [48]. GMA was first polymerized, followed by MMA. An aliquot of the living diblock copolymer was picked out, deactivated by methanol and analyzed by SEC and ${ }^{1} \mathrm{H}$ NMR. Two equivalents of a difunctional monomer (i.e. ethyleneglycol bismethacrylate) were then added to the living diblock macroanions in order to form the star-shaped block copolymer.

Linear and star-shaped diblocks were analyzed by size exclusion chromatography (SEC) and proton nuclear magnetic resonance $\left({ }^{1} \mathrm{H}\right.$ NMR). SEC was carried out in tetrahydrofuran, with a Hewlett-Packard 1050 liquid chromatograph equipped with two PL gel columns (1000 and $10,000 \AA$, respectively) and a Hewlett-Packard 1047A refractive index detector.

Poly(methylmethacrylate) standards were used for calibration. ${ }^{1} \mathrm{H}$ NMR spectra were recorded at $400 \mathrm{MHz}$ with the Bruker AM 400 spectrometer. $M_{\mathrm{n}}$ of the second block was calculated from the ${ }^{1} \mathrm{H}$ NMR spectrum of the copolymer and $M_{\mathrm{n}}$ of the first block. The apparent mean number of arms $(U)$ in the star-shaped diblock was estimated from the ratio of the molecular weights for the 'arm' and the star-shaped diblock, respectively. In this calculation, $M_{\mathrm{n}}$ for the star was approximated by SEC, which leaves some uncertainty on $U$. This apparent number of arms was found close to 2 , which suggests preferential formation of a triblock copolymer. 
The PGMA block was selectively sulfonated by ring-opening of the oxirane pendent groups by sodium sulfite [44-45]. Sodium sulfite $(50 \mathrm{~g})$ and tetrabutylammonium bromide (transfer catalyst, $33 \mathrm{~g}$ ) were dissolved in $200 \mathrm{ml}$ of water in a flask equipped with a condenser. The finely divided PMMA- $b$-PGMA copolymer (20 g) was suspended in the stirred aqueous solution, and $20 \mathrm{ml}$ of chloroform were finally added. The suspension was deoxygenated by bubbling of nitrogen and heated at $80^{\circ} \mathrm{C}$ for $30 \mathrm{~h}$. Known amounts of PMMA- $b$-PSGMA copolymers were dissolved in water and purified by dialysis against regularly replaced distilled water (Spectra-Por membranes, cut-off 10,000 Da). The degree of sulfonation was determined by titration and was found to be ca. $70 \%$ for all the samples. The molecular characteristics of all the copolymers analyzed in this study are listed in Table 1.

Table 1. Molecular characteristic features of the samples considered in this study

\begin{tabular}{|c|c|c|c|c|c|c|c|c|}
\hline \multirow[t]{2}{*}{ Acronym } & PMMA & & Comolyn & & \multirow[t]{2}{*}{ PGMA $M_{a}$} & \multirow[t]{2}{*}{ Sulfonation degee (is) } & \multicolumn{2}{|l|}{ PSGMA } \\
\hline & & $M_{\mathrm{w}} M_{\mathrm{a}}$ & $M_{n}$ & $M_{w} / M_{0}$ & & & $M_{a}$ & $w s$ \\
\hline PMIA(d.1)-b- & 4100 & 1.05 & 20,500 & 1.10 & 16,400 & 70 & 24700 & 86 \\
\hline $\begin{array}{l}\text { PSGMA }(11.5)- \\
b \text {-PMMA }(4.8)-b-\end{array}$ & 4800 & 1.65 & 19.500 & 1.25 & $15,200^{\circ}$ & 68 & $23,060^{\circ}$ & 83 \\
\hline $\begin{array}{l}\text { FSGMA(11.5) } \\
\text { PMMA(13.1)-b- } \\
\text { PSGMAi } 22.7)\end{array}$ & 13.100 & 1.10 & 28.400 & 1.10 & 15.300 & 67 & 22.700 & 63 \\
\hline
\end{tabular}

\subsection{Preparation of the copolymer solutions}

All the solutions were prepared by dilution of the dialyzed copolymer solution by bidistilled water.

\subsection{Surface tension measurements}

Surface tensions were measured at $20^{\circ} \mathrm{C}$ with a Kruss tensiometer and a calibrated platinum plate. The reproducibility was ascertained by frequent checking of the surface tension of bidistilled water (surface tension=72-73 $\mathrm{mN} / \mathrm{m}$ ).

\subsection{Dynamic light scattering (DLS)}

DLS measurements were carried out with a Brookhaven Instruments. DLS apparatus that consists of a BI-200 goniometer, a BI-2030 digital correlator and an Ar-ion laser (LEXEL Lasers) with a wavelength of $488 \mathrm{~nm}$. The scattering angle used for the measurements was $90^{\circ}$. A refractive index matching bath of filtered decalin surrounded the scattering cell, and the temperature was controlled at $25^{\circ} \mathrm{C}$. Prior to sample loading, appropriate glass vessels were soaked overnight in sulfochromic solution, thoroughly cleaned with bidistilled water and dried in a vacuum oven.

The experimental correlation function $G_{2}(t)$ was measured. For a single exponential decay, $G_{2}(t)$ can be expressed by Eq. (1)

$G_{2}(t)=B[1+\beta \exp (-2 \Gamma t)] \mathbf{( 1 )}$

where $B$ is the baseline, $\beta$ an optical constant that depends on the instrument, $t$ the time and $\Gamma$ the decay rate for the process which is given by

$\Gamma=D q^{2}$ 
where $D$ is the translation diffusion coefficient, and $q$ the absolute value of the scattering vector

$q=[4 \pi n \sin (\theta / 2)] / \lambda(3)$

where $n$ is the refractive index of the solvent, $\theta$ the diffusion angle and $\lambda$ the wavelength of the incident light.

The diffusion coefficient extrapolated to zero concentration $\left(D_{0}\right)$ for spherical particles is related to the hydrodynamic radius, $R_{\mathrm{h}}$, by the Stokes-Einstein equation:

$D_{0}=k_{\mathrm{B}} T / 6 \pi \eta R_{\mathrm{h}}$

where $k_{\mathrm{B}}$ is the Boltzmann constant, $T$ the absolute temperature and $\eta$ the viscosity of the solvent.

When aggregates of different sizes were formed in solution, the experimental correlation function depends on all the individual decay processes. In this case, the data were analyzed by the Contin routine, a constrained regularization method program for the inverse Laplace transformation of DLS data. The Contin program gave access to the distribution of the relaxation times in the experimental time correlation functions. $Z$-averaged distribution of $R_{\mathrm{h}}$ was then calculated.

\subsection{Size exclusion chromatography of the amphiphiles}

The eluent was an aqueous solution of $\mathrm{NaNO}_{3}(0.1 \mathrm{~mol} / \mathrm{l})$, tris(hydroxymethyl)aminoethane $(0.025 \mathrm{~mol} / \mathrm{l})$ and EDTA $(0.001 \mathrm{~mol} / \mathrm{l})$. The $\mathrm{pH}$ of the eluent was adjusted to 9 with $\mathrm{HCl}$. A Waters model 600E liquid chromatograph was equipped with a TosoHaas TSKgel G4000 $\mathrm{PW}_{\mathrm{xl}}$ and a UV detector (Waters 486). The elution rate was $0.5 \mathrm{ml} / \mathrm{min}$, and the copolymer concentration was $5 \mathrm{~g} / 1$.

\section{Results and discussion}

Three PMMA- $b$-PSGMA copolymers were synthesized and characterized (Table 1). They are designated as PMMA $(x)-b$-PSGMA $(y)$, where $x$ and $y$ are $M_{\mathrm{n}} \times 10^{-3}$ of each block. The PSGMA block is kept basically unchanged in these three samples, whereas the hydrophobic PMMA block is varied. The PSGMA(11.5)- $b$-PMMA(4.8)- $b$-PSGMA(11.5) sample can, indeed, be considered as the triblock analog of the PMMA(4.1)- $b$-PSGMA(24.7) diblock of a comparable composition.

The surface tension of the aqueous solutions of the copolymers was measured in order to determine the critical micelle concentration $(\mathrm{cmc})$. The average size and the size distribution of the micelles were measured by DLS. Finally, the effect of temperature on the aggregation of the copolymers was studied by DLS and SEC. All the copolymers were readily dissolved in water and purified by dialysis, so leading to bluish scattering aqueous solutions. This preparation method of the copolymer solutions is basically different from that one used in previous works. Indeed, Selb and Gallot observed micellization of quaternized poly(4vinylpyridine)-block-polystyrene in methanol/water/salt mixtures. The copolymers were first dissolved in methanol and then added with the aqueous electrolyte solution [24]. When the water content of the solution was increased, the average degree of the copolymer association 
passed through a maximum. Similarly, Munk et al. studied poly(methacrylic acid)-blockpolystyrene copolymers and the formation of micelles with a polystyrene core in water [2935]. These copolymers could not be directly dissolved in water but first in dioxane/water mixtures rich in dioxane. The micelles formed in this solvent mixture were transferred in pure water or in aqueous buffers by stepwise dialysis. Tuzar et al. showed that the size of these micelles increased with the dioxane content of the mixed solvent, more likely as result of the swelling of the polystyrene core [35]. Baines et al. also used a non-selective organic solvent in the preliminary dissolution step of poly(methyl methacrylate)-block-poly(dimethylaminoethyl methacrylate) copolymers, thus prior to micellization in water [36].

In a previous study, we showed that low molecular weight poly(methyl methacrylate)-blockpoly(dimethylaminoethyl methacrylate) copolymers that contained a major hydrophilic block $(>70 \mathrm{wt} \%)$ could be directly dissolved in water, with formation of micelles thought to be close to the thermodynamic equilibrium [38]. In this paper, no organic cosolvent was used to prepare the micellar solutions.

\subsection{Surface tension measurement}

A decrease in the cmc with the hydrophobic content is usually observed for amphiphilic block copolymers in aqueous solution. Indeed, the $\mathrm{cmc}$ of Pluronics was found to decrease exponentially when the length of the poly(propylene oxide) block was increased, whatever the hydrophilic block length was [49-50]. In contrast, an increase in the length of the poly(ethylene oxide) block at constant hydrophobic block length resulted in a slightly higher cmc [51]. The same conclusions held on for polystyrene-block-poly(acrylic acid) diblocks. Indeed, the cmc considerably decreased when the polystyrene block length was increased, at a constant polyelectrolyte block length [52]. In contrast, changing the soluble block length at constant hydrophobic block length had only a small effect on the cmc [52].

The surface tension $(\gamma)$ was plotted against the copolymer concentration for all the samples considered in this study (Fig. 1). All the samples show the typical behavior of surfactants, which allows the cmc to be determined as the concentration at the intersection of the tangents to the curves (Fig. 1 and Table 2). Because the micellization is observed nearly at the same concentration for the three copolymers under consideration, no clear conclusion on the dependence of the cmc on the copolymer composition can be drawn. The cmc's of the PMMA(4.1)- $b$-PSGMA(24.7) and PSGMA(11.5)- $b$-PMMA(4.8)- $b$-PSGMA(11.5) copolymers of approximately the same composition are slightly different, which suggests a small effect of the block copolymer architecture (di- vs. triblock).

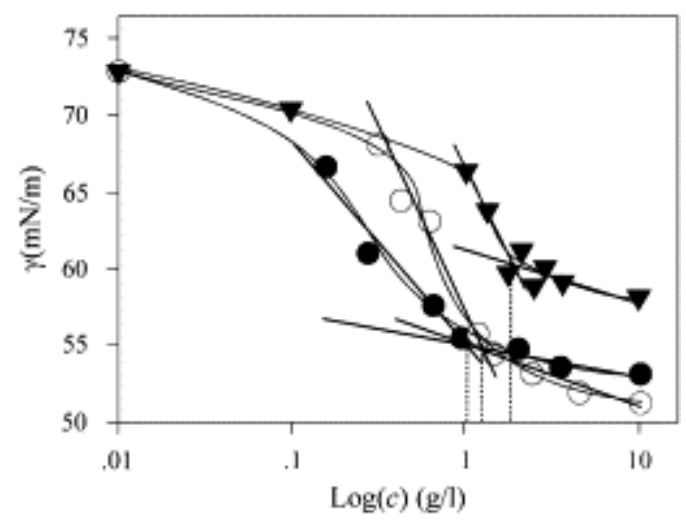


Fig. 1. Surface tension ( $\gamma$ ) as a function of the copolymer concentration $(C)$ in water at $25^{\circ} C$ : (black triangles) PMMA(13.1)-b-PSGMA(22.7); (open dots) PSGMA(11.5)-b-PMMA(4.8)-bPSGMA(11.5); (black dots) PMMA(4.1)-b-PSGMA(24.7).

Table 2. Surfactant properties of the copolymers

\begin{tabular}{lccccc}
\hline Acronym & $\mathrm{cmc}(\mathrm{g} /)^{\mathrm{a}}$ & $\mathrm{cmc}(\mathrm{g} /)^{\mathrm{b}}$ & $\Pi_{\mathrm{mm}}(\mathrm{mN} / \mathrm{m})$ & $\sigma\left(\mathrm{A}^{2} / \mathrm{molecule}\right)$ & $\Delta G_{\text {min }}^{0}(\mathrm{~kJ} / \mathrm{mol})^{c}$ \\
\hline PMMA(4.1)-b- & $1=0.2$ & $1=0.1$ & 54 & 69 & -25.0 \\
PSGMA(24.7) & $1.2 \pm 0.3$ & $1.4 \pm 0.2$ & 52 & 46 & -34.5 \\
PSGMA(11.5)- & & & & & \\
$b-\operatorname{PMMA}(4.3)-b-$ & $2.3 \pm 0.2$ & $2.2 \pm 0.3$ & 59 & 43 & -23.5 \\
PSGMA(11.5) & & & & & \\
PMMA(13.1)-b- & & & & \\
PSGMA(22.7) & & &
\end{tabular}

Some information about the thermodynamics of micellization can be extracted from the $\mathrm{cmc}$, on the assumption that the association is closed. The equilibrium constant $\left(K_{\mathrm{C}}\right)$ of micellization can then be written as

$K_{\mathrm{C}}=\left[\mathrm{M}_{N}\right]^{1 / N} /[\mathrm{M}](5)$

where $\mathrm{M}$ designates individual copolymer chains and $\mathrm{M}_{N}$ refers to micelles that contain $N$ copolymer chains. If $N>50$, then the equilibrium constant can be approximated by

$K_{\mathrm{C}} \quad 1 /[\mathrm{M}](6)$

where $[\mathrm{M}]$ is nothing but the cmc. Therefore, the free enthalpy of micellization, $\Delta G_{\text {mic }}{ }^{0}$, can be calculated as follows:

$\Delta G_{\text {mic }}^{0}=R T \ln (\mathrm{cmc})$

This calculation is valid to an ideal solution of copolymer chains in the standard conditions. The calculated $\Delta G_{\text {mic }}{ }^{0}$ values are reported in Table 2 and found essentially independent of the PMMA- $b$-PSGMA samples considered in this study. $N$ was assumed to be higher than 50 for the micelles formed by the three copolymers under consideration.

The area occupied per copolymer molecule at the air/water interface $(\sigma)$ has been calculated, according to Eq. (8)

$\sigma=R T / \Pi N_{\mathrm{A}}(8)$

where $N_{\mathrm{A}}$ is the Avogadro's number and $\Pi$ the surface pressure calculated from Eq. (9), thus from the slope of the surface tension vs. the logarithm of the surfactant concentration just below the cmc:

$\Pi=[\mathrm{d} \gamma / \mathrm{d} \ln C]_{T, C<\mathrm{cmc}}(9)$

The data in Table 2 clearly show that the conformation of the copolymer chains at the air/water interface changes considerably with the samples under consideration. Indeed, the PMMA(4.1)-b-PSGMA(24.7) molecules occupy a larger area at the air/water interface compared to the two other samples. Although the PMMA(13.1.)- $b$-PSGMA(22.7) sample is of a higher molecular weight, its area at the air/water interface is lower. 
The influence of the molecular architecture is highlighted by the comparison of the PSGMA(11.5)-b-PMMA(4.8)- $b$-PSGMA(11.5) and PMMA(4.1)- $b$-PSGMA(24.7) samples which have nearly the same total molecular weight and composition, but occupy a considerably different area at the air/water interface. According to data in Table 2, the PSGMA(11.5)- $b$-PMMA(4.8)-b-PSGMA(11.5) chains are more extensively stretched at the air/water interface than the diblock chains. This situation is thought to result from the very close proximity of the two PSGMA(11.5) blocks, which are connected to each other by the short PMMA(4.8) block.

Finally, the area occupied at the air/water interface by each copolymer can be compared to the cmc. For all the samples under consideration, the lower the $\mathrm{cmc}$ is, the higher area at the air/water interface is. This observation is consistent with the approximation according to which the cmc can be approximated to the concentration of the copolymer chains at the air/water interface.

\subsection{Dynamic light scattering measurements}

Aggregation has also been studied by DLS measurements. In this discussion, structures with a size of a few tens of nanometers will be referred to as micelles, and larger structures will be designated as aggregates, following the terminology commonly used in the scientific literature $[5-8 ; 40 ; 42]$. Unassociated copolymer chains are called unimers. The mean hydrodynamic radii are very large, which indicates that the micelles are at least partly aggregated. Indeed, a Contin analysis of the experimental correlation function shows one population of well-defined aggregates for the PMMA(13.1)-b-PSGMA(22.7) sample (Fig. 2a), a very broad distribution of the aggregate size for the PMMA(4.1)- $b$-PSGMA(24.7) sample (Fig. 2b) and two distinct populations of aggregates for the PSGMA(11.5)- $b$-PMMA(4.8)- $b$-PSGMA(11.5) sample (Fig. $2 \mathrm{c})$. These aggregates result either from the association of several micelles into larger structures or from the incomplete dissolution of the copolymer. In the latter case, the time needed to reach the equilibrium should be exceedingly long, because the glassy PMMA core prevents exchange between unimers and micelles from occurring and well-defined micelles from being formed. In this respect, the unimers-micelles exchange rate is negligible at room temperature in case of polystyrene-block-poly(ethylene oxide) diblocks [54]. In contrast, the exchange rate is ca. $10^{-3} \mathrm{~s}^{-1}$ for poly(sodium methacrylate)-blockpoly(dimethylaminoethylmethacrylate) diblocks, in line with the $T_{\mathrm{g}}$ of the hydrophobic aminated block which is close to room temperature [55]. 
a)

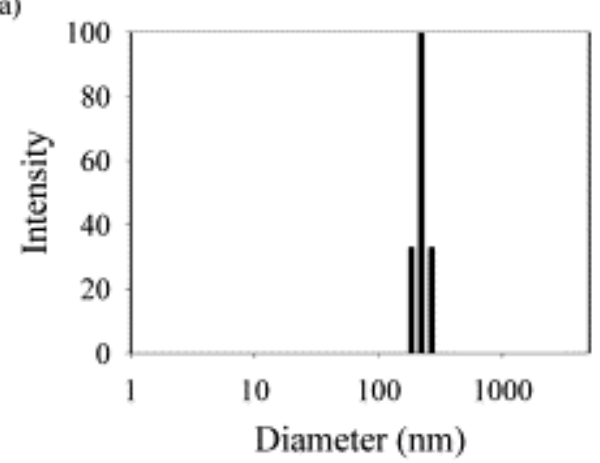

b)

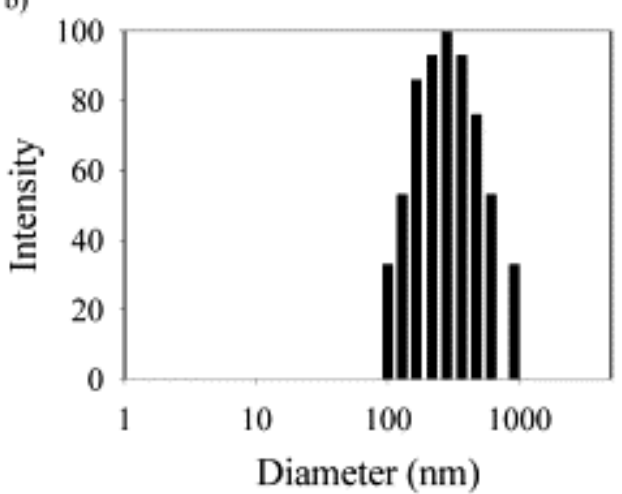

c)

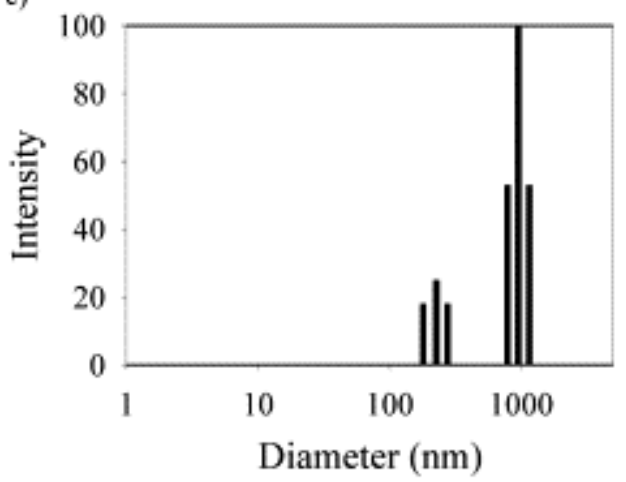

Fig. 2. Contin size distribution of the particles formed in water at room temperature: (a) PMMA(13.1)-b-PSGMA(22.7); (b) PMMA(4.1)-b-PSGMA(24.7); (c) PSGMA(11.5)-bPMMA(4.8)-b-PSGMA(11.5).

The scattered intensity has been plotted as a function of the logarithm of the copolymer concentration for all the samples under investigation (Fig. 3). Upon decreasing copolymer concentration, a sharp drop in the scattered intensity is systematically observed before a plateau is reached. The transition between these two distinct regimes actually corresponds to the $\mathrm{cmc}$, which is in agreement with the data reported from the surface tension measurements. 


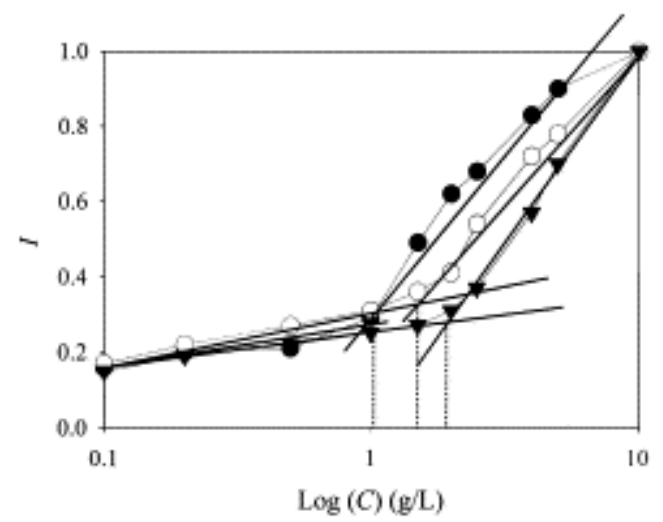

Fig. 3. Normalized scattered intensity (I) as a function of the copolymer concentration: (black triangles) PMMA(13.1)-b-PSGMA(22.7); (open dots) PSGMA(11.5)-b-PMMA(4.8)-bPSGMA(11.5); (black dots) PMMA(4.1)-b-PSGMA(24.7).

\subsection{Effect of temperature on the aggregation}

The effect of temperature has been studied in order to dissociate the large structures observed by DLS at room temperature. The change in $R_{\mathrm{h}}$ has been monitored up to $80^{\circ} \mathrm{C}$ for all the samples. Fig. 4 shows that the average $R_{\mathrm{h}}$ decreases as the temperature is increased for the PMMA(4.1)- $b$-PSGMA(24.7) and PSGMA(11.5)-b-PMMA(4.8)- $b$-PSGMA(11.5) copolymers, whereas it remains constant in the case of the PMMA(13.1)- $b$-PSGMA(22.7) sample for which, however, $R_{\mathrm{h}}$ was much lower at $25^{\circ} \mathrm{C}$. Another interesting observation is the evolution of the Contin histograms with temperature, as shown in Fig. 5 for the PMMA(4.1)- $b$-PSGMA(24.7) diblock and in Fig. 6 for the PSGMA(11.5)- $b$-PMMA(4.8)- $b$ PSGMA(11.5) triblock. Upon increasing temperature, at least two, if not three populations of particles are observed, and their relative importance changes in favor of the smallest particles. In sharp contrast, the Contin histogram of the PMMA(13.1)-b-PSGMA(22.7) sample is essentially independent of temperature, at least up to $75^{\circ} \mathrm{C}$. This observation suggests that the aggregates formed by this copolymer are very stable, consistently with glassy PMMA cores, whose glass transition temperature exceeds the upper limit of the temperature range scanned in this study. It must be recalled that $Z$-averaged data are made available by the Contin routine. It is indeed well known that the scattered intensity of a particle is related to its size and can be roughly approximated to the cubic power of the aggregate molecular weight. Therefore, $Z$-averaged data need to be calculated if populations of different sizes have to be compared in terms of number of aggregates.

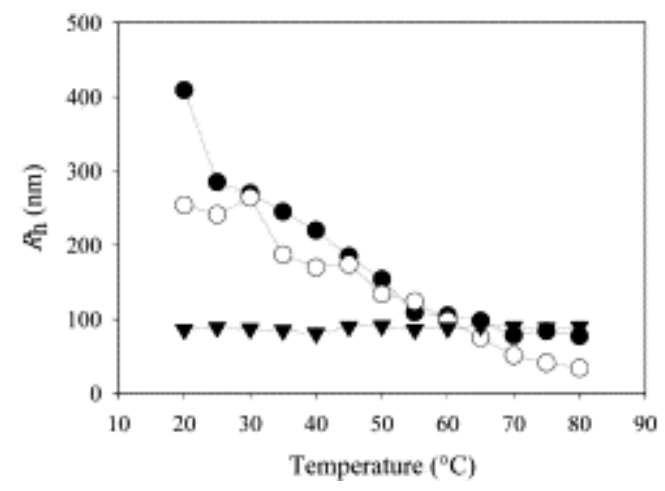


Fig. 4. $R_{h}$ as a function of temperature: (black triangles) PMMA(13.1)-b-PSGMA(22.7); (open dots) PSGMA(11.5)-b-PMMA(4.8)-b-PSGMA(11.5); (black dots) PMMA(4.1)-bPSGMA(24.7).
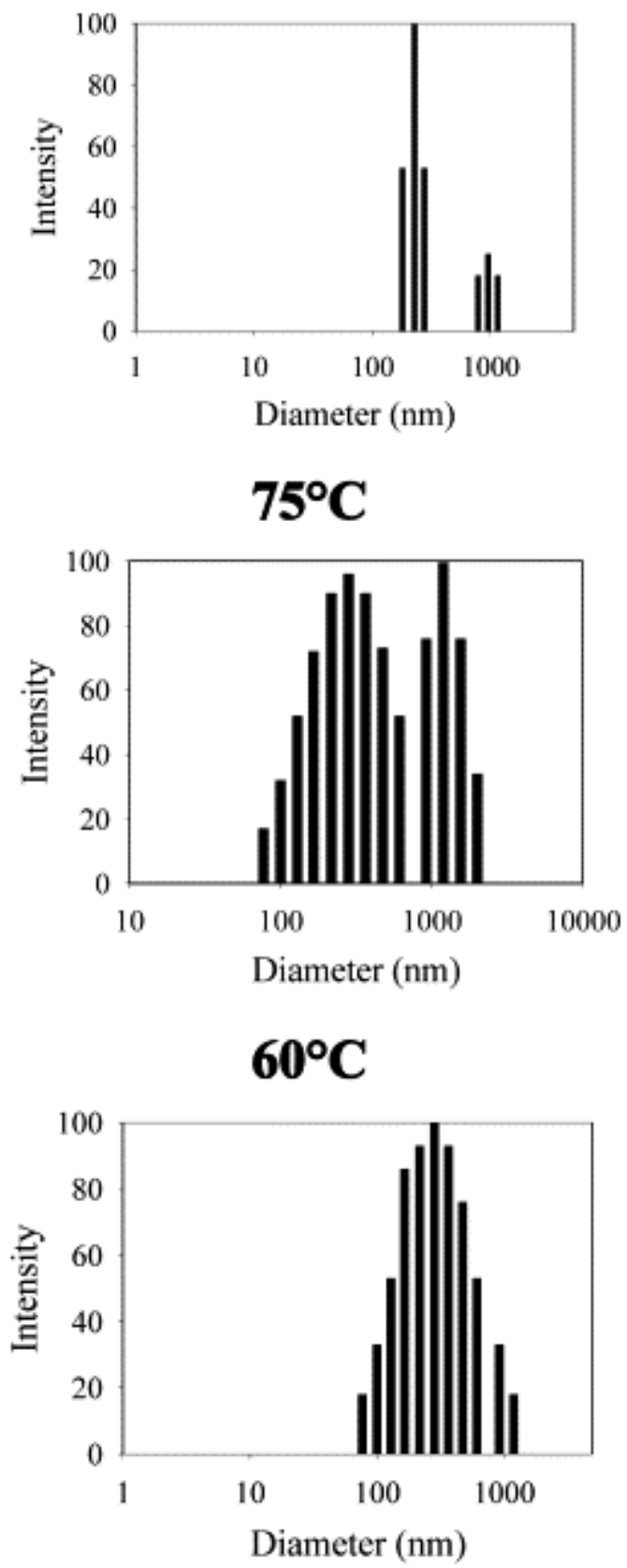

\section{$40^{\circ} \mathrm{C}$}

Fig. 5. Contin size distribution for the particles formed by the PMMA(4.1)-b-PSGMA(24.7) diblock in water at three different temperatures. 


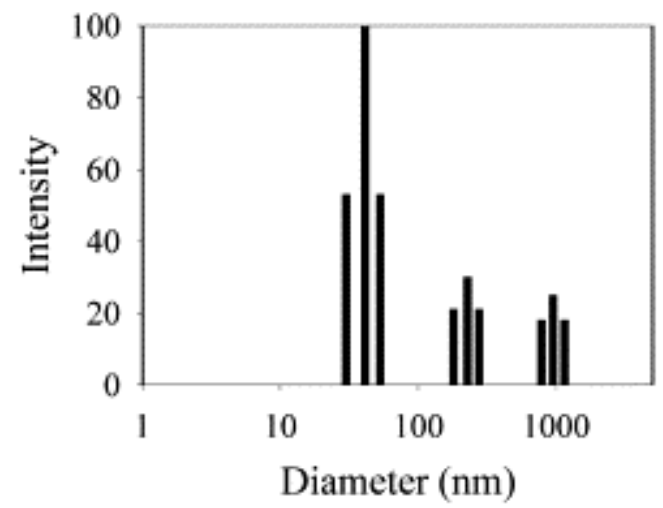

$7^{\circ} \mathrm{C}$

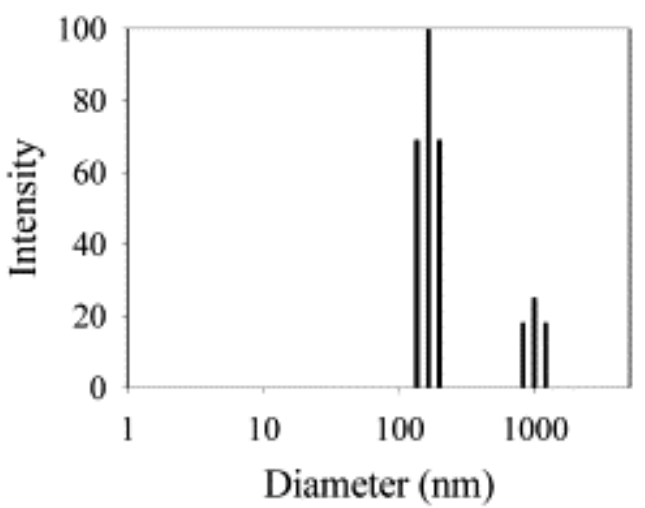

\section{$60^{\circ} \mathrm{C}$}

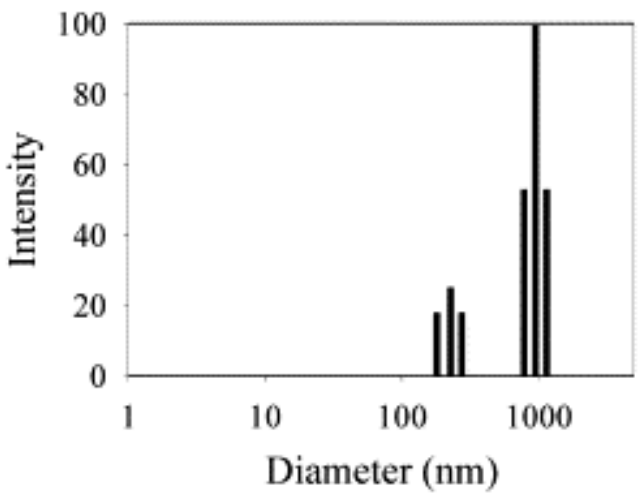

\section{$40^{\circ} \mathrm{C}$}

Fig. 6. Contin size distribution for the particles formed by the PSGMA(11.5)-b-PMMA(4.8)-bPSGMA(11.5) diblock in water at three different temperatures.

According to Fig. 5, the broad size distribution of the aggregates formed by the PMMA(4.1)$b$-PSGMA(24.7) diblock at $25^{\circ} \mathrm{C}$ is split in two distinct populations at higher temperature, and the smaller size population dominates at $75^{\circ} \mathrm{C}$. These particles have, however, sizes larger than micelles, which indicates that the primary micelles are not released yet at $75^{\circ} \mathrm{C}$.

When the PSGMA(11.5)- $b$-PMMA(4.8)- $b$-PSGMA(11.5) triblock is concerned, Fig. 6 shows that a third population emerges at $75^{\circ} \mathrm{C}$, with a size which is now consistent with micelles. 
Therefore, when the molecular weight of the hydrophobic PMMA block is low, i.e. in the $4000-5000$ range, the glass transition $\left(T_{\mathrm{g}}\right)$ of the PMMA cores is low enough $\left(T_{\mathrm{g}} \text { at ca. } 70^{\circ} \mathrm{C}\right)^{1}$ to allow the copolymer chains to reorganize, which is no longer the case when the PMMA block is ca. three times longer $\left(13,000, T_{\mathrm{g}}\right.$ of the PMMA core at ca. $\left.115^{\circ} \mathrm{C}\right) .{ }^{1}$ It thus appears that a short PMMA hydrophobic block is a prerequisite to observe changes in the aggregates/micelles partitioning as the temperature is increased.

All these observations have been confirmed by SEC. Indeed, Fig. 7, Fig. 8 and Fig. 9 show several elution peaks that can be assigned to unimers, micelles and aggregates [56-57], respectively. Peaks observed at the lower elution times (ca. 9-14 min) are the signature of the more or less big aggregates, whereas unimers are eluted at the larger time (ca. $20 \mathrm{~min}$ ). Consistently, the elution time for a PSGMA(19.5) homopolymer is ca. $20 \mathrm{~min}$. It should be noted that unimers are detected by SEC, which is not the case by DLS due to experimental limits. Moreover, the solvent which flows through the SEC columns has a shear effect that could modify the unimers/micelles/aggregates partitioning. Therefore, the SEC and DLS experiments can only be compared in a qualitative way. Nevertheless, there is a fairly good agreement between the evolution of the Contin size distribution and the SEC traces with temperature for the three copolymers under consideration. Indeed, temperature has no effect on the aggregation of the PMMA(13.1)- $b$-PSGMA(22.7) diblock, as shown in Fig. 7 and discussed above. The same trends in the evolution of the PMMA(4.1)- $b$-PSGMA(24.7) and the PSGMA(11.5)- $b$-PMMA(4.8)- $b$-PSGMA(11.5) samples with temperature are observed by DLS and SEC. In agreement with the DLS data (Fig. 5 and Fig. 6), different types of aggregates are observed for these two samples by SEC in the 9-12 min elution range. The peak observed at ca. 17 min of elution at $75^{\circ} \mathrm{C}$ (Fig. 9) for the PSGMA(11.5)- $b$-PMMA(4.8)$b$-PSGMA(11.5) copolymer is consistent with micelles, when compared to the DLS data reported in Fig. 6.

\footnotetext{
${ }^{1}$ The glass transition temperature of the PMMA core was measured by differential scanning calorimetry (TA Instruments 2010 apparatus, heating rate $10^{\circ} \mathrm{C} / \mathrm{min}$ ).
} 


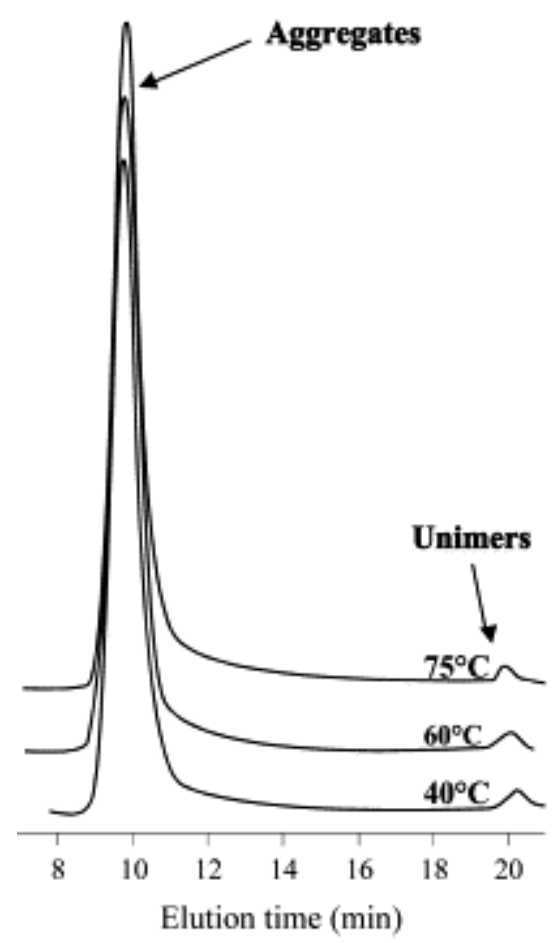

Fig. 7. SEC traces for the PMMA(13.1)-b-PSGMA(22.7) diblock in water at three different temperatures.

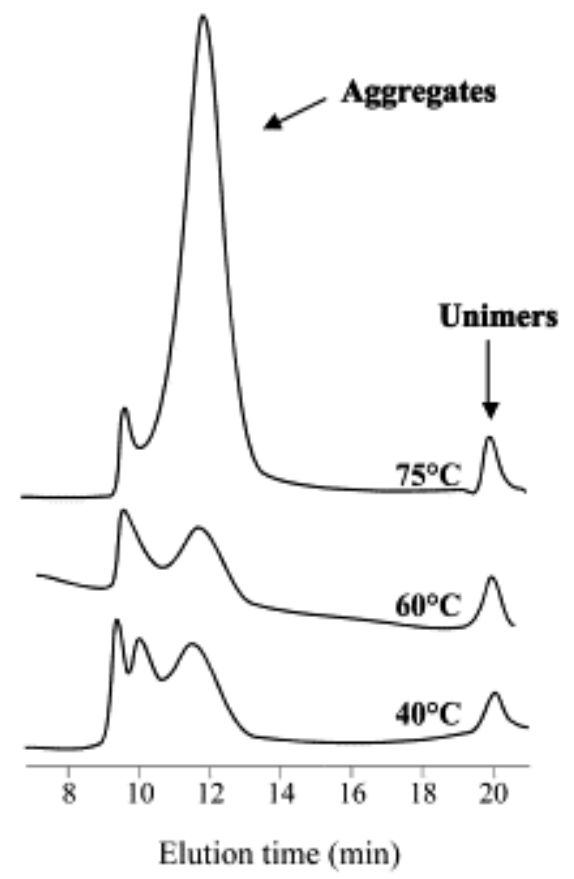

Fig. 8. SEC traces for the PMMA(4.1)-b-PSGMA(24.7) diblock in water at three different temperatures. 


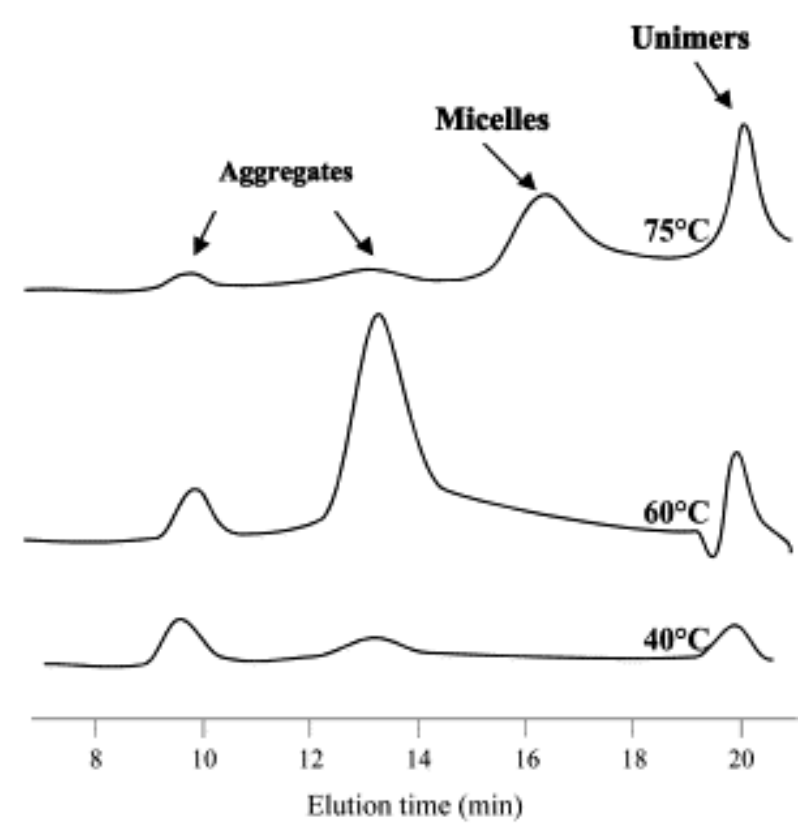

Fig. 9. SEC traces for the PSGMA(11.5)-b-PMMA(4.8)-b-PSGMA(11.5) diblock in water at three different temperatures.

Finally, the reversibility of the aggregation has been checked by slowly cooling the copolymer solution $\left(1{ }^{\circ} \mathrm{C} / \mathrm{min}\right)$ down to room temperature. Fig. 10 compares the temperature dependence of $R_{\mathrm{h}}$ for the PSGMA(11.5)- $b$-PMMA(4.8)-b-PSGMA(11.5) triblock, upon heating and cooling, respectively. The reversibility of the copolymer aggregation in water is confirmed, although some hysteresis may be noted. This observation supports that the aggregates observed in water do not result from the incomplete dissolution of the copolymer. The same conclusion holds for the PMMA(4.1)- $b$-PSGMA(24.7) diblock.

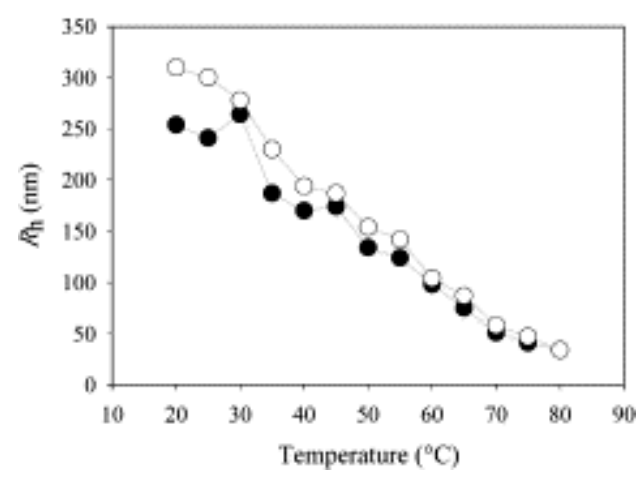

Fig. 10. $R_{h}$ as a function of temperature for the PSGMA(11.5)-b-PMMA(4.8)-b-PSGMA(11.5) triblock in water (open dots: cooling cycle, black dots: heating cycle).

\section{Conclusions}

In this paper, the aggregation of three PMMA- $b$-PSGMA copolymers has been studied. These samples show a characteristic cmc and thus behave as polymeric surfactants. Although these copolymers form micelles above a critical concentration, these micelles tend to associate into larger structures (aggregates). The copolymers containing the shorter hydrophobic blocks are the most effective surfactants. The aggregation equilibrium of these copolymers is shifted towards micelles or at least smaller aggregates upon increasing the temperature. In sharp 
contrast, the aggregates formed by the sample which contains the longer hydrophobic block are insensitive to temperature in the $25-75^{\circ} \mathrm{C}$ range. The monitoring of the aggregation at different temperatures by DLS and SEC is in qualitative agreement.

\section{References}

[1] Yokoyama M, Inoue S, Kataoka K, Yui N, Sakurai Y. Makromol Chem Rapid Commun $1987 ; 8: 431$.

[2] Yokoyama M, Okano T, Sakurai Y, Ekimoto H, Shibazaki C, Kataoka K. Cancer Res 1991;51:3229.

[3] Creutz S, Jérôme R, Kaptijn GMP, van der Wert AW, Akkerman JM. J Coat Techol 1998;70(883):41.

[4] Winzor CL, Mrazek Z, Winnik MA, Croucher RD, Riess D. Eur Polym J 1994;30:121.

[5] Tuzar Z, Krachtovil P. In: Matijevic E, editor. Surface and colloid science. New York: Plenum Press, 1993.

[6] Chu B. Langmuir 1995;11:414.

[7] Alexandridis P. Curr Opin Colloid Interf Sci 1996;1:490.

[8] Moffit M, Khougaz K, Eisenberg A. Ace Chem Res 1996;29:95.

[9] Bedells AD, Arafeh RM, Yang Z, Attwood D, Collet JH, Price C, Booth C. J Chem Soc, Faraday Trans 1993;89:1235.

[10] Luo YZ, Nicholas CV, Attwood D, Collet JH, Price C, Booth C, Chu B, Zhou ZK. J Chem Soc, Faraday Trans 1993;89:539.

[11] Nicholas CV, Luo YZ, Deng NJ, Attwood D, Collet JH, Price C, Booth C. Polymer 1993;34:138.

[12] Alexandridis P, Athanassiou V, Fukuda S, Hatton TA. Langmuir 1994; 10:2604.

[13] Yang L, Bedells AD, Attwood D, Booth C. J Chem Soc, Faraday Trans 1992;88:1447.

[14] Deng Y, Ding J, Stubbersfield RB, Heatley F, Attwood D, Price C, Booth C. Polymer 1992;33:1963.

[15] Brown W, Schillen K, Almgren M, Hvidt S, Bahadur P. J Phys Chem 1991;95:1850.

[16] Malmsten M, Lindman B. Macromolecules 1992;25:5440.

[17] Almgren M, Bahadur P, Jansson M, Li P, Brown W, Bahadur A. J Colloid Interf Sci 1992; 151:157.

[18] Linse P. Macromolecules 1993;26:4437.

[19] Mortensen K, Brown W. Macromolecules 1993;26:4128.

[20] Almgren M, Brown W, Hvidt S. Colloid Polym Sci 1995;273:2.

[21] Wilhem M, Zhao CL, Wang Y, Xu R, Winnik MA, Mura JL, Riess G, Croucher MD. Macromolecules 1991;24:1033.

[22] Hruska Z, Piton M, Yekta A, Duhamel J, Winnik MA, Riess G, Croucher MD.

Macromolecules 1993;26:1825.

[23] Selb J, Gallot Y. Makromol Chem 1980;181:809.

[24] Selb J, Gallot Y. Makromol Chem 1980;181:2605.

[25] Selb J, Gallot Y. Makromol Chem 1981;182:1491.

[26] Selb J, Gallot Y. Makromol Chem 1981;182:2523.

[27] Biggs S, Vincent B. Colloid Polym Sci 1992;270:563.

[28] Gao ZS, Varshney SK, Wong S, Eisenberg A. Macromolecules 1994;27:7923.

[29] Tuzar Z, Webber SE, Ramireddy C, Munk P. Polym Prepr 1991;32:525.

[30] Cao T, Munk P, Ramireddy C, Tuzar Z, Webber SE. Macromolecules 1991;24:6300.

[31] Kiserow D, Prochazka K, Ramireddy C, Tuzar Z, Munk P, Webber SE. Macromolecules 1992;25:461. 
[32] Prochazka K, Kiserow D, Ramireddy C, Webber SE, Munk P, Tuzar Z. Makromol Chem Macromol Symp 1992;58:201.

[33] Chan J, Fox S, Kiserow D, Ramireddy C, Munk P, Webber SE. Macromolecules 1993;26:7016.

[34] Tian M, Qin A, Ramireddy C, Webber SE, Munk P, Tuzar Z, Prochakza K. Langmuir 1993;9:1741.

[35] Tuzar Z, Kratochvil P, Prochakza K, Munk P. Collect Czech Chem Commun 1993;58:2362.

[36] Baines FL, Armes SP, Billingham NC, Tuzar Z. Macromolecules 1996;29:8151.

[37] Baines FL, Billingham NC, Armes SP. Macromolecules 1996;29:3416.

[38] Antoun S, Gohy JF, Jérôme R. Polymer 2001;42:3641.

[39] Selb J, Gallot Y. In: Goodman I, editor. Developments in block copolymers, vol. 2.

Amsterdam: Elsevier, 1985. p. 85.

[40] Zhang L, Eisenberg A. Science 1995;268:1728.

[41] Discher BM, Won YY, Ege DS, Lee JCM, Bates FS, Discher DE, Hammer DA. Science 1999;284:1143.

[42] Yu K, Eisenberg A. Macromolecules 1998;31:3509.

[43] Gohy JF, Creutz S, Garcia M, Mahltig B, Stamm M, Jérôme R. Macromolecules 2000;33:6378.

[44] Leemans L, Fayt R, Teyssié Ph. J Polym Sci 1990;28:1255.

[45] Leemans L, Fayt R, Teyssié Ph, de Jaeger NC. Macromolecules 1991;24:5922.

[46] Abraham T, Giasson S, Gohy JF, Jérôme R. Langmuir 2000; 16:4286.

[47] Abraham T, Giasson S, Gohy JF, Jérôme R, Miiller B, Stamm M. Macromolecules 2000;33:6051.

[48] Zilliox JG, Rempp P, Parrod J. J Polym Sci, Part C 1968;22:145.

[49] Alexandridis P, Holzwarth JF, Hatton TA. Macromolecules 1994;27:2414.

[50] Wanka G, Hoffmann M, Ulbricht W. Macromolecules 1994;27:4145.

[51] Chu B, Zhou Z. Nonionic surfactants. In: Nace VN, editor. Polyoxyalkylene block copolymers, vol. 60. New York: Marcel Dekker, 1996.

[52] Astafieva I, Zhong XF, Eisenberg A. Macromolecules 1993;26:7339.

[54] Wang Y, Balaji R, Quirk RP, Mattice WL. Polym Bull 1992;28:333.

[55] Creutz S, van Stam J, Antoun S, De Schryver F, Jérôme R. Macromolecules 1997;30:4078.

[56] Creutz S, van Stam J, De Schryver F, Jérôme R. Macromolecules 1998;31:681.

[57] Wang QG, Price C, Booth C. J Chem Soc, Faraday Trans 1992;88:1437. 